\title{
Three-dimensional topological twistronics
}

\author{
Fengcheng Wu $\bullet$, Rui-Xing Zhang, and Sankar Das Sarma \\ Condensed Matter Theory Center and Joint Quantum Institute, Department of Physics, University of Maryland, \\ College Park, Maryland 20742, USA
}

(Received 12 October 2019; accepted 24 March 2020; published 13 April 2020)

\begin{abstract}
We introduce a theoretical framework for the concept of three-dimensional (3D) twistronics by developing a generalized Bloch band theory for 3D layered systems with a constant twist angle $\theta$ between successive layers. Our theory employs a nonsymmorphic symmetry that enables a precise definition of an effective out-of-plane crystal momentum, and also captures the in-plane moiré pattern formed between neighboring twisted layers. To demonstrate topological physics that can be achieved through 3D twistronics, we present two examples. In the first example of chiral twisted graphite, Weyl nodes arise because of inversion-symmetry breaking, with $\theta$-tuned transitions between type-I and type-II Weyl fermions, as well as magic angles at which the in-plane velocity vanishes. In the second example of a twisted Weyl semimetal, the twist in the lattice structure induces a chiral gauge field $\mathcal{A}$ that has a vortex-antivortex lattice configuration. Line modes bound to the vortex cores of the $\mathcal{A}$ field give rise to 3D Weyl physics in the moiré scale. We also discuss possible experimental realizations of 3D twistronics.
\end{abstract}

DOI: 10.1103/PhysRevResearch.2.022010

Introduction. Moiré superlattices formed in twisted bilayers lead to interesting two-dimensional (2D) phenomena. In twisted bilayer graphene (TBG), there are magic twist angles, at which moiré bands become nearly flat due to a vanishing Dirac velocity [1] and many-body interactions are effectively enhanced. TBG represents a prototypical system for 2D twistronics [2], where the twist angle serves as a tuning parameter in controlling material properties. Given the greatly exciting 2D physics developing in TBG such as the discovery of superconducting and correlated insulating states [3-14], it is natural to wonder whether the concept of twistronics can be generalized to $3 \mathrm{D}$ systems.

In this Rapid Communication, we present a theoretical framework for 3D twistronics that can be realized in $3 \mathrm{D}$ layered systems with a constant twist angle $\theta$ between successive layers. This 3D chiral twisted structure [Fig. 1(a)] generally breaks the translational symmetry in all spatial directions and thus the conventional Bloch theorem cannot be applied. However, the structure has an exact nonsymmorphic symmetry, which consists of an in-plane $\theta$ rotation followed by an out-of-plane translation. We use this screw rotational symmetry to define a generalized Bloch's theorem, where the modified crystal momenta are well defined. Various 3D moiré physics can be explored by considering different 2D building blocks in our theoretical framework.

We apply our theory to two systems. In the first system of chiral twisted graphite with graphene as the 2D building block, Weyl fermions arise due to the inversion-symmetry

Published by the American Physical Society under the terms of the Creative Commons Attribution 4.0 International license. Further distribution of this work must maintain attribution to the author(s) and the published article's title, journal citation, and DOI. breaking in the twisted structure. Both type-I and type-II [15] Weyl fermions can be realized depending on the value of $\theta$. Moreover, we find two magic angles at which the in-plane Fermi velocity of the Weyl fermions vanishes, representing the realization of magic-angle Weyl physics. In the second system of a twisted Weyl semimetal, we study the effects of chiral twist in the lattice structure on Weyl fermions that already exist even without the twist. The chiral twist induces a chiral gauge field $\mathcal{A}$ that has a vortex-antivortex lattice configuration in the moiré pattern formed between adjacent twisted layers. The vortex cores of the $\mathcal{A}$ field bind line modes with position-dependent chiralities, which generalizes the quasi-1D physics of a Weyl nanotube under torsion [16] to 3D. The periodic array of the coupled vortex line modes gives rise to 3D Weyl fermions with moiré-scale modulations in the wave function. Therefore, the twist angle provides another tuning knob to create and manipulate Weyl fermions, and, more generally, topological phases in 3D.

Theory. We construct a generalized Bloch band theory for the chiral twisted structure shown in Fig. 1(a). The continuum Hamiltonian for this system is

$$
\begin{aligned}
H= & \sum_{n} \int d^{2} \boldsymbol{r}\left\{\psi_{n}^{\dagger}(\boldsymbol{r}) h_{n}\left(\boldsymbol{k}_{\|}\right) \psi_{n}(\boldsymbol{r})\right. \\
& \left.+\left[\psi_{n}^{\dagger}(\boldsymbol{r}) T_{n}(\boldsymbol{r}) \psi_{n+1}(\boldsymbol{r})+\text { H.c. }\right]\right\},
\end{aligned}
$$

where $n$ is the layer index, $\boldsymbol{r}$ and $\boldsymbol{k}_{\|}=-i \partial_{\boldsymbol{r}}$ are respectively the $2 \mathrm{D}$ in-plane position and momentum operators, $\psi_{n}^{\dagger}(\boldsymbol{r})$ represents the field operator for low-energy states, $h_{n}\left(\boldsymbol{k}_{\|}\right)$is the in-plane Hamiltonian for each 2D building block, and $T_{n}(\boldsymbol{r})$ is the interlayer tunneling. Here, $\psi^{\dagger}$ can be a multicomponent spinor due to sublattices, orbitals, spins, etc. The layer dependence of $h_{n}$ and $T_{n}$ is determined by the twist relation

$$
h_{n}\left[\hat{R}(n \theta) \boldsymbol{k}_{\|}\right]=h_{0}\left(\boldsymbol{k}_{\|}\right), \quad T_{n}[\hat{R}(n \theta) \boldsymbol{r}]=T_{0}(\boldsymbol{r}),
$$



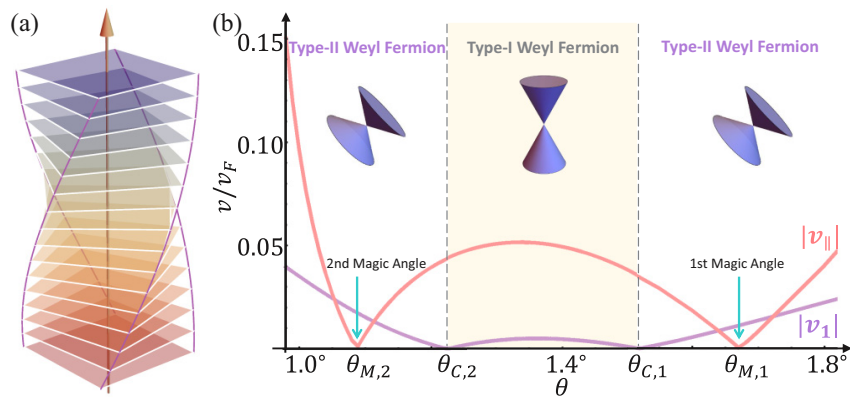

FIG. 1. (a) Illustration of a 3D twisted structure with a constant twist angle $\theta$ between successive layers. (b) Summary of results on magic-angle Weyl fermions in chiral twisted graphite. The plot shows the in-plane velocity $v_{\|}$and one of the out-of-plane velocities $v_{1}$ for the Weyl fermion at $\boldsymbol{k}_{1 / 2}=(0,0, \pi / 2) . v_{\|}$vanishes at magic angles $\theta_{M, 1}$ and $\theta_{M, 2} . v_{1}$ changes sign at $\theta_{C, 1}$ and $\theta_{C, 2}$, which mark transitions between type-I and type-II Weyl fermions.

where $\hat{R}$ is a rotation matrix. $T_{0}(\boldsymbol{r})$ has an in-plane moiré periodicity $(\propto 1 / \theta)$ when $\theta$ is small.

The 3D twisted structure generally breaks translational symmetry in all spatial directions, making it appear hopeless for theoretical treatments. However, Eq. (2) implies that the Hamiltonian $H$ is invariant under a nonsymmorphic operation, which rotates a layer by $\theta$ and then translates it along the out-of-plane $\hat{z}$ direction by the interlayer distance $d_{z}$. This nonsymmorphic symmetry suggests a generalized Bloch wave for the system,

$$
\psi_{k_{z}}(\boldsymbol{r})=\frac{1}{\sqrt{N}} \sum_{n} e^{-i n k_{z}} \psi_{n}[\hat{R}(n \theta) \boldsymbol{r}],
$$

where $N$ is the number of layers, and the good quantum number $k_{z}$ is an effective out-of-plane crystal momentum measured in units of $1 / d_{z}$. This Bloch wave is a superposition of electron states on a spiral line around the screw-rotation axis, as illustrated by the purple lines in Fig. 1(a). Under this generalized Bloch representation, the Hamiltonian $H$ becomes

$$
\begin{aligned}
H= & \sum_{k_{z}} \int d^{2} \boldsymbol{r}\left\{\psi_{k_{z}}^{\dagger}(\boldsymbol{r}) h\left(\boldsymbol{k}_{\|}\right) \psi_{k_{z}}(\boldsymbol{r})\right. \\
& \left.+\left[\psi_{k_{z}}^{\dagger}(\boldsymbol{r}) e^{i k_{z}} T(\boldsymbol{r}) \psi_{k_{z}}[\hat{R}(-\theta) \boldsymbol{r}]+\text { H.c. }\right]\right\},
\end{aligned}
$$

where we use $h$ and $T$ as shorthand notations respectively for $h_{0}$ and $T_{0}$. It is worth noting that the appearance of $\hat{R}(-\theta)$ in Eq. (4) signals the breaking of in-plane translation symmetries. To proceed, we expand $T(\boldsymbol{r})$ by moiré harmonics, $T(\boldsymbol{r})=\sum_{g} T_{g} e^{i g \cdot \boldsymbol{r}}$, where $\boldsymbol{g}$ is a moiré reciprocal lattice vector. $T(\boldsymbol{r})$ generates in-plane momentum scatterings specified by $\boldsymbol{k}_{\|}^{\prime}=\hat{R}(\theta) \boldsymbol{k}_{\|}+\boldsymbol{g}$. For low-energy physics, $\left|\boldsymbol{k}_{\|}\right|$is generally of the same order of magnitude as $|\boldsymbol{g}|$, which is proportional to $\theta$. Thus, $\hat{R}( \pm \theta)$ can be approximated by an identity matrix in the small $\theta$ limit, and the error is on the order $\theta|\boldsymbol{g}| \ll|\boldsymbol{g}|$. Under this approximation, $H$ acquires a moiré translational symmetry,

$$
\begin{aligned}
H & \approx \sum_{k_{z}} \int d^{2} \boldsymbol{r} \psi_{k_{z}}^{\dagger}(\boldsymbol{r})\left[h\left(\boldsymbol{k}_{\|}\right)+\Delta\left(k_{z}, \boldsymbol{r}\right)\right] \psi_{k_{z}}(\boldsymbol{r}), \\
\Delta\left(k_{z}, \boldsymbol{r}\right) & =e^{i k_{z}} T(\boldsymbol{r})+e^{-i k_{z}} T^{\dagger}(\boldsymbol{r}),
\end{aligned}
$$

which gives rise to energy bands in the 3D momentum space spanned by $k_{z}$ and the in-plane moiré Brillouin zone. Equation (5) is our effective Hamiltonian for the 3D small-angle twisted system, which builds in exactly the nonsymmorphic symmetry and captures the moiré pattern formed in neighboring twisted layers.

Chiral twisted graphite. We apply our theory to study the electronic structure of chiral twisted graphite, which we construct by starting from an infinite number of graphene layers with $A A A \ldots$ stacking, and then rotating the $n$th layer by $n \theta$ around a common hexagon center. In each layer, low-energy electrons reside in $\pm K$ valleys, which are related by spinless time-reversal symmetry $\hat{\mathcal{T}}$ and can be studied separately as in TBG. We focus on the $+K$ valley, with the in-plane $\boldsymbol{k} \cdot \boldsymbol{p}$ Hamiltonian $h\left(\boldsymbol{k}_{\|}\right)=\hbar v_{F} \boldsymbol{k}_{\|} \cdot \boldsymbol{\sigma}$, where $v_{F}$ is the monolayer graphene Dirac velocity $\left(\sim 10^{6} \mathrm{~m} / \mathrm{s}\right)$ and $\sigma$ is the sublattice Pauli matrix. The interlayer tunneling $T(\boldsymbol{r})$ is $[1,17]$

$$
T(\boldsymbol{r})=\sum_{j=0,1,2}\left(\begin{array}{cc}
w_{A A} & w_{A B} e^{-i 2 \pi j / 3} \\
w_{A B} e^{i 2 \pi j / 3} & w_{A A}
\end{array}\right) e^{i \boldsymbol{g}_{j+1} \cdot \boldsymbol{r}},
$$

where $w_{A A}$ and $w_{A B}$ are respectively the intrasublattice and intersublattice tunneling parameters, with $w_{A A} \approx 90 \mathrm{meV}$ and $w_{A B} \approx 117 \mathrm{meV} . g_{1}$ is a moiré reciprocal lattice vector $\left(0,4 \pi / 3 a_{M}\right)$, and $a_{M}=a_{0} / \theta$, where $a_{0}$ is the monolayer graphene lattice constant. The other two vectors $\boldsymbol{g}_{2,3}$ are related to $g_{1}$ by $\pm 2 \pi / 3$ rotations. We note that $a_{M}$ is the TBG moiré periodicity, but $T(\boldsymbol{r})$ in Eq. (6) has a periodicity of $\tilde{a}_{M}=\sqrt{3} a_{M}$.

The $k_{z}$-dependent moiré potential $\Delta=e^{i k_{z}} T+e^{-i k_{z}} T^{\dagger}$ can be decomposed into $\Delta_{0} \sigma_{0}+\Delta_{x} \sigma_{x}+\Delta_{y} \sigma_{y}$, where $\Delta_{0}$ is a scalar potential. From $\Delta$, we can define an effective gauge field $\mathcal{A}=\left(\Delta_{x}, \Delta_{y}\right) /\left(e v_{F}\right)$ that couples to the Dirac Hamiltonian $h\left(\boldsymbol{k}_{\|}\right)$, and a corresponding pseudomagnetic field $b_{z}=$ $\nabla_{r} \times \mathcal{A}$. 2D maps of $\Delta_{0}$ and $b_{z}$ at $k_{z}=0$ are plotted in Fig. 2, which shows that $\left|b_{z}\right|$ can reach $\sim 200 \mathrm{~T}$ for $\theta=1.1^{\circ}$.

The effective Hamiltonian $\mathcal{H}=h\left(\boldsymbol{k}_{\|}\right)+\Delta$ respects $\hat{C}_{3 z}$ and $\hat{C}_{2 z} \hat{\mathcal{T}}$ symmetries, where $\hat{C}_{n z}$ is the $n$-fold rotation around $\hat{z}$ axis. We diagonalize $\mathcal{H}$ using a plane-wave expansion, and show the calculated band structures at $\theta=1.1^{\circ}$ in Fig. 2. Bands along the $k_{z}$ axis can be characterized by the $\hat{C}_{3 z}$ angular momentum $\ell_{z} \in\{0, \pm 1\}$. As shown in Fig. 2(d), crossings between two bands with different $\ell_{z}$ actually represent 3D Weyl nodes, which appear abundantly along the $k_{z}$ axis. For example, the Weyl fermion at the $\gamma$ point $(\boldsymbol{k}=\mathbf{0})$ has an effective Hamiltonian $\hbar\left(v_{F}^{*} \boldsymbol{k}_{\|} \cdot \boldsymbol{\sigma}+v_{z}^{*} k_{z} \sigma_{z}\right)$, which is constrained by both $\hat{C}_{3 z}$ and $\hat{C}_{2 z} \hat{\mathcal{T}}$ symmetries. The $\theta$ dependence of $\left(v_{F}^{*}, v_{z}^{*}\right)$ is shown in Fig. 2(c). $v_{F}^{*}$ is reduced from the bare value $v_{F}$, but remains finite for $\theta$ from $0.7^{\circ}$ to $2^{\circ}$. Remarkably, the sign of $v_{z}^{*}$ oscillates with $\theta$, and therefore the chirality of the Weyl node at the $\gamma$ point is twist angle dependent.

Another representative Weyl node is located at $\boldsymbol{k}_{1 / 2}=$ $(0,0, \pi / 2)$. This Weyl node is pinned to zero energy by a particle-hole-like symmetry $\mathcal{H}\left(-\boldsymbol{k}_{\|}, \pi-k_{z},-\boldsymbol{r}\right)=$ $-\mathcal{H}\left(\boldsymbol{k}_{\|}, \boldsymbol{k}_{z}, \boldsymbol{r}\right)$, and is described by $\hbar\left[v_{\|} \boldsymbol{k}_{\|} \cdot \boldsymbol{\sigma}+v_{1} q_{z}\left(\sigma_{0}+\right.\right.$ $\left.\left.\sigma_{z}\right) / 2+v_{2} q_{z}\left(\sigma_{0}-\sigma_{z}\right) / 2\right)$ ], where $v_{1,2}$ are two independent parameters and $q_{z}=k_{z}-\pi / 2$. For $\theta$ between $1^{\circ}$ and $1.8^{\circ}$, $v_{2}$ is always negative, but $v_{1}$ changes sign at $\theta_{C, 1} \approx 1.52^{\circ}$ and $\theta_{C, 2} \approx 1.22^{\circ}$, which are critical angles that mark the transitions between type-I and type-II Weyl fermions [Fig. 1(b)]. 

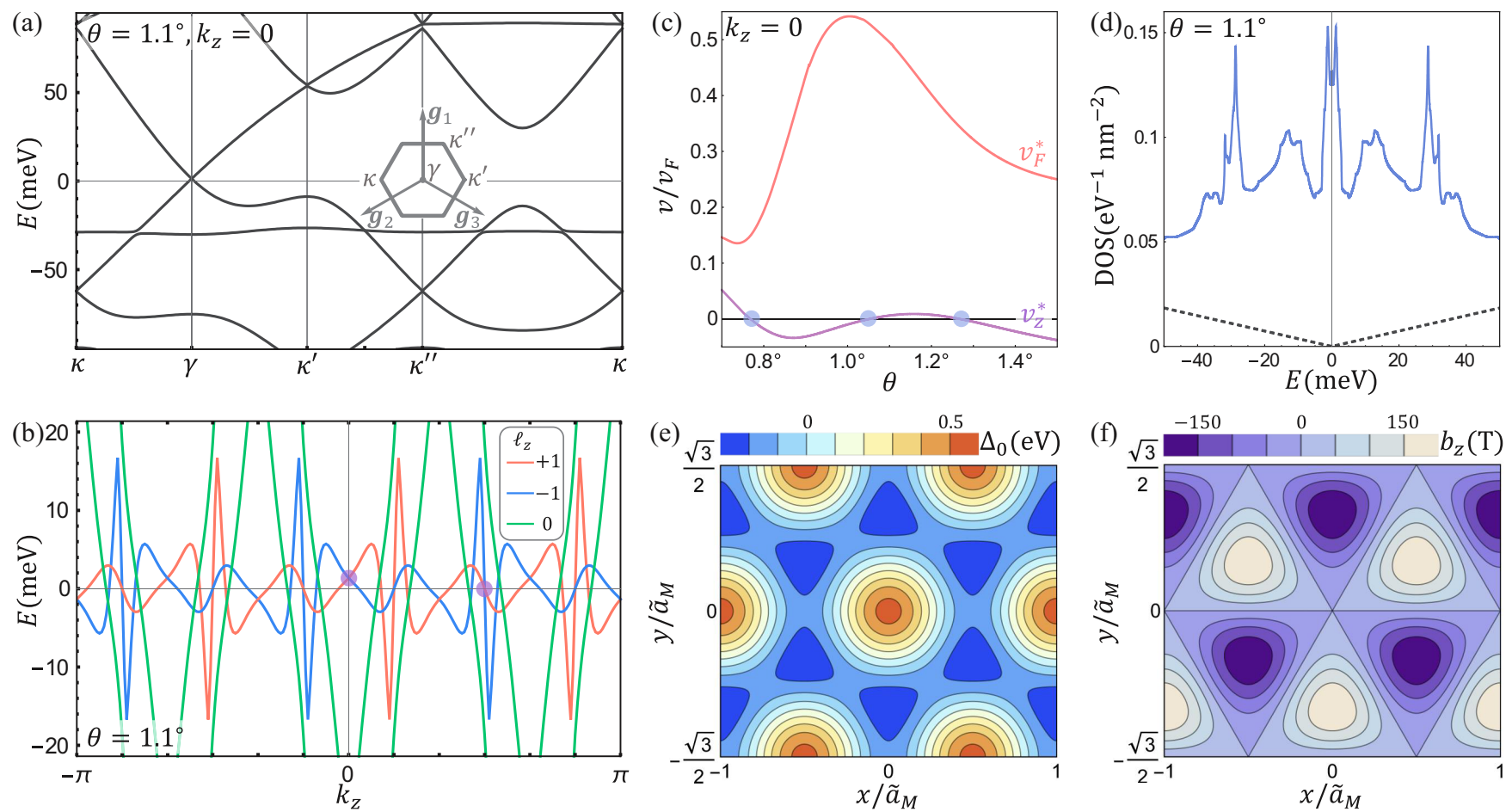

FIG. 2. Results of chiral twisted graphite. (a) In-plane and (b) out-of-plane band structure. In (a), $k_{z}$ is 0 . In (b), $\boldsymbol{k}_{\|}$is zero, the crossings between bands with different $\ell_{z}$ represent Weyl nodes, the two purple dots highlight nodes located at $k_{z}=0$ and $k_{z}=\pi / 2$, and the spectrum is periodic in $k_{z}$ with a period $2 \pi / 3$ due to a translationlike symmetry $\mathcal{H}\left(\boldsymbol{k}_{\|}, k_{z}+2 \pi / 3, \boldsymbol{r}+a_{M} \hat{y}\right)=\mathcal{H}\left(\boldsymbol{k}_{\|}, k_{z}, \boldsymbol{r}\right)$. (c) In-plane and out-of-plane velocities of the $\boldsymbol{k}=\mathbf{0}$ Weyl node as a function of $\theta$. (d) DOS per spin, valley, layer, and area for the twisted graphite (blue line), and corresponding DOS for monolayer graphene (black dashed line). (e) 2D maps of the scalar moiré potential $\Delta_{0}$ and (f) pseudomagnetic field $b_{z}$ at $k_{z}=0$ and $\theta=1.1^{\circ}$.

Moreover, the in-plane velocity $v_{\|}$vanishes at both $\theta_{M, 1} \approx$ $1.67^{\circ}$ and $\theta_{M, 2} \approx 1.09^{\circ}$, which can be identified as two magic angles. Here, the value of $\theta_{M, 1}$ can also be estimated using an analytical perturbation theory, agreeing quantitatively with our direct band-structure calculations; see Supplemental Material (SM) [18]. The low-energy density of states (DOS) per layer in the twisted graphite near the magic angles is orders of magnitude larger than that in monolayer graphene [Fig. 2(d)], which should enhance the interaction effects, leading to interaction-driven quantum phase transitions.

We now compare our results with related works. Reference [19] studied multiple graphene layers with a twist angle $(-1)^{n} \theta$ that alternates with the layer index $n$. Their 3D structure preserves inversion symmetry, in contrast with our chiral twisted graphite structure. Reference [20] studied the same structure as ours but with a different method under the coherent phase approximation. Our theory employs the exact nonsymmorphic symmetry, which allows us to precisely define the $k_{z}$ momentum and clearly demonstrate magic-angle Weyl physics in the twisted graphite. In Ref. [21], twisted trilayer graphene has been theoretically studied. An interesting question is how many layers are required in practice to realize our predicted 3D physics, which we leave for future study.

Twisted Weyl semimetal. As another demonstration, we apply our theory to a twisted Weyl semimetal with a lattice structure also shown in Fig. 1(a). We start by introducing a minimal Weyl semimetal model on a simple cubic lattice (lattice constant $\left.a_{0}\right), h_{W}=\hbar v_{W} \boldsymbol{k}_{\|} \cdot \boldsymbol{\sigma}+M(\boldsymbol{k}) \sigma_{z}$, where $M(\boldsymbol{k})=$
$M_{0}\left(\cos k_{z}-\cos Q_{z}\right)-M_{1} \boldsymbol{k}_{\|}^{2}$ with parameters $M_{0,1}>0$ and $0<Q_{z}<\pi$. Each site on the cubic lattice accommodates two orbitals $\left|j_{z}=\frac{3}{2}\right\rangle$ and $\left|j_{z}=\frac{1}{2}\right\rangle$, which are labelled by the angular momentum $j_{z}$ and form the basis of the Pauli matrices $\sigma$. The Hamiltonian $h_{W}$ breaks time-reversal symmetry and hosts two Weyl nodes with opposite chiralities located respectively at $\boldsymbol{k}_{ \pm}=\left(0,0, \pm Q_{z}\right)$.

We now consider a twisted cubic lattice in which the $n$th layer is rotated by $n \theta$ around the $\hat{z}$ axis following Fig. 1(a). The effective Hamiltonian $\mathcal{H}_{W}$ for this twisted structure is given by

$$
\mathcal{H}_{W}=h_{W}+2 t_{s p} \sin k_{z} \sum_{g}^{\prime}\left(\begin{array}{cc}
0 & e^{-i\left(\phi_{g}+g \cdot r\right)} \\
e^{i\left(\phi_{g}+g \cdot r\right)} & 0
\end{array}\right),
$$

where $t_{s p}$ characterizes the interorbital tunneling between neighboring layers. The summation over $\boldsymbol{g}$ is restricted to the first shell of moiré reciprocal lattice vectors $\left(|\boldsymbol{g}|=2 \pi / a_{M}\right.$ with the moiré period $a_{M}$ equal to $\left.a_{0} / \theta\right)$, and $\phi_{g}$ is the orientation angle of $\boldsymbol{g}$. Here, the interlayer tunneling matrix is derived using a two-center approximation, and the spatial modulation of interlayer intraorbital tunneling is neglected, which are thoroughly explained in the SM [18]. Compared with the twisted graphite Hamiltonian, $\mathcal{H}_{W}$ does not have a scalar moiré potential term.

We can extract an effective gauge field $\mathcal{A}$ from $\mathcal{H}_{W}$, similar to the case of twisted graphite. The $\mathcal{A}$ field has a vortexantivortex lattice configuration in the moiré superlattices [18]. 

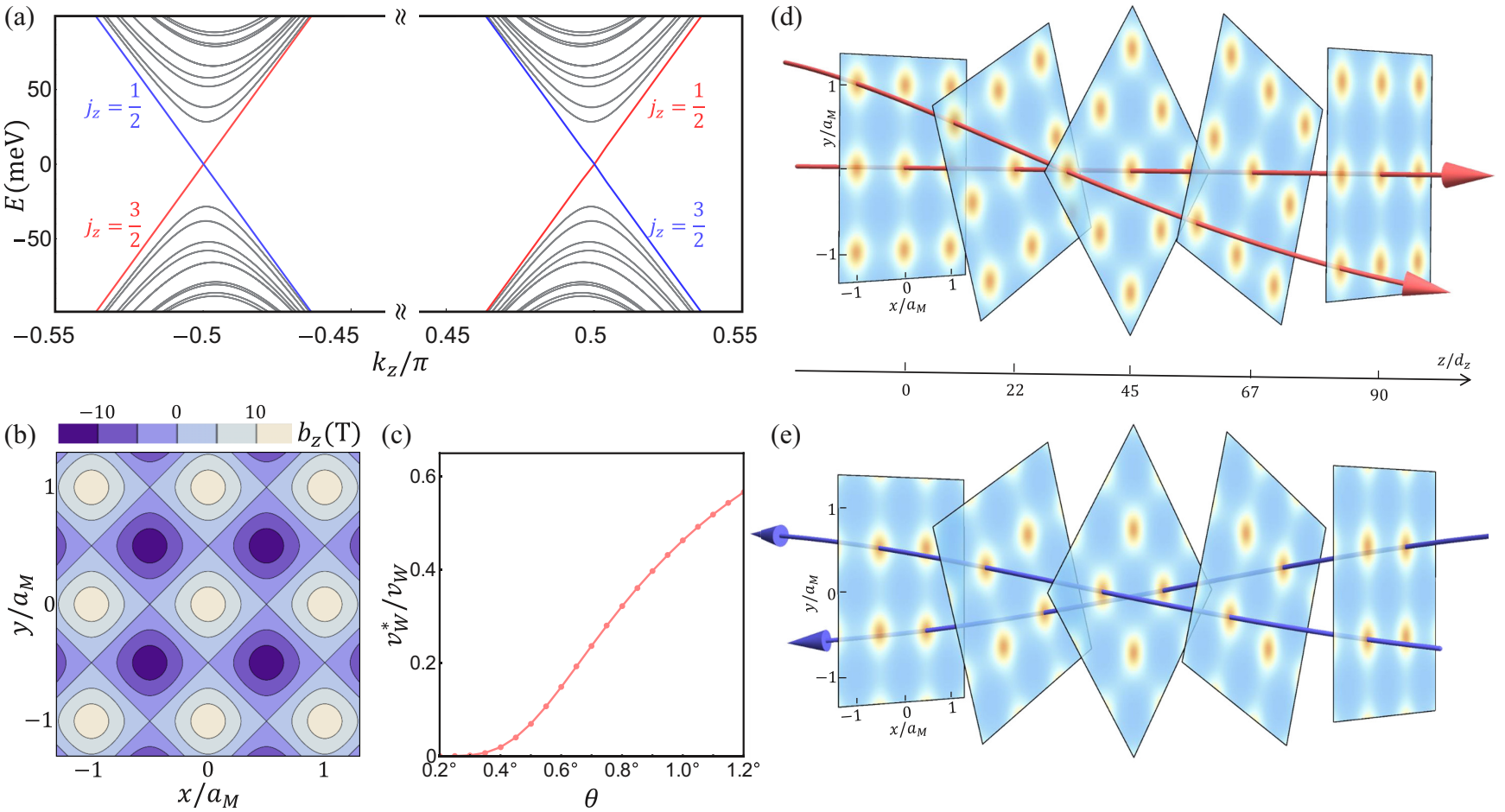

FIG. 3. Results of twisted Weyl semimetal. (a) Out-of-plane band structure in the twisted Weyl semimetal. In-plane momentum is zero. Red and blue lines respectively mark right- and left-moving modes that cross Weyl nodes located at $k_{z}= \pm \pi / 2 . j_{z}$ specifies the angular momentum of each mode. Model parameters are $M_{0}=869 \mathrm{meV}, M_{1}=10.36 \mathrm{eV} \AA^{2}, t_{s p}=4 \mathrm{meV}, v_{W}=3.74 \times 10^{5} \mathrm{~m} / \mathrm{s}, a_{0}=7.5 \AA$, and $Q_{z}=\pi / 2$. (b) 2D maps of the pseudomagnetic field $b_{z}\left(k_{z}=\pi / 2\right)$, which is positive and negative, respectively, around integer $\mathcal{R}$ and half-integer $\boldsymbol{\mathcal { R }}_{1 / 2}$ positions. $\theta=1^{\circ}$ in (a) and (b). (c) The in-plane velocity $v_{W}^{*}$ of the Weyl node as a function of $\theta$. The out-of-plane velocity $v_{z}^{*}$ (not shown) barely changes with $\theta$. (d) Real-space probability density of the right-moving modes in (a). The peaks of the density are at $\mathcal{R}$ positions in each 2D layer, and wind around the screw rotation axis. (e) Similar as (d) but for the left-moving modes in (a), and the peaks are located at $\boldsymbol{\mathcal { R }}_{1 / 2}$ positions.

The corresponding pseudomagnetic field $b_{z}$ is given by

$$
b_{z}=\frac{2 t_{s p}}{e v_{W}} \frac{2 \pi}{a_{M}} \sin k_{z} \sum_{g}^{\prime} e^{i \boldsymbol{g} \cdot \boldsymbol{r}},
$$

which is illustrated in Fig. 3(b). $\left|b_{z}\right|$ peaks at two distinct types of positions in the moiré pattern, namely, integer positions $\mathcal{R}=\left(n_{x}, n_{y}\right) a_{M}$ with $n_{x, y} \in \mathbb{Z}$ and half-integer positions $\mathcal{R}_{1 / 2}=\mathcal{R}+\left(\frac{1}{2}, \frac{1}{2}\right) a_{M}$, which are also locations of vortex cores of the $\mathcal{A}$ field. We assume $t_{s p} / v_{W}>0$, and $b_{z}$ is positive and negative respectively at $\mathcal{R}$ and $\mathcal{R}_{1 / 2}$ for $0<k_{z}<\pi$. Being proportional to $\sin k_{z}, b_{z}$ represents a chiral magnetic field as it couples oppositely to the Weyl nodes with different topological charges. In the semiclassical picture, around $\mathcal{R}$ $\left(\boldsymbol{\mathcal { R }}_{1 / 2}\right)$ positions, this chiral magnetic field leads to chiral Landau levels that propagate along the positive (negative) $\hat{z}$ direction for both Weyl nodes at $\boldsymbol{k}_{ \pm}$. These chiral Landau levels bound to vortex cores of the $\mathcal{A}$ field can be considered to be vortex line modes (VLMs). The chirality of a VLM depends on its position in the moiré pattern. An important physical consequence is that an out-of-plane electric field can drive a real-space pumping of electrons from $\mathcal{R}$ to $\boldsymbol{R}_{1 / 2}$ positions, or vice versa. The 2D array of VLMs can further hybridize with each other, and we expect them to realize Weyl fermions with moiré-scale modulations in the wave functions.
To verify the above picture, we numerically diagonalize $\mathcal{H}_{W}$, and show the energy spectrum along $k_{z}$ in Fig. 3(a), where we find that the Weyl nodes are robust against the twist. In Fig. 3(d) [Fig. 3(e)], we plot the real-space probability density of the right- (left-) moving modes highlighted in Fig. 3(a), which is found to be primarily concentrated at $\mathcal{R}\left(\mathcal{R}_{1 / 2}\right)$ positions. This density profile is consistent with the above semiclassical analysis. Because of the twisted structure, these modes track spiral lines in real space. From the semiclassical picture, the in-plane velocity $v_{W}^{*}$ of the 3D Weyl fermions is controlled by the coupling strength between neighboring VLMs, and therefore by the moiré period $a_{M}$. Numerical results plotted in Fig. 3(c) confirm that $v_{W}^{*}$ decreases with decreasing $\theta$ (equivalently, increasing $a_{M}$ ). For small enough $\theta, v_{W}^{*}$ nearly vanishes, showing that VLMs located at different positions are essentially decoupled. Thus, the twist angle provides an alternative tuning knob to control the band structure of 3D Weyl materials.

Conclusion. In summary, we develop a general theoretical framework for 3D twistronics and apply the theory to chiral twisted graphite and twisted Weyl semimetal. Our theory can in principle be realized in van der Waals heterostructures constructed by stacking multiple twisted layers. Moreover, chiral twisted van der Waals nanowires have recently been experimentally synthesized [22,23], indicating that the chiral twisted structure we study can indeed appear in materi- 
als. Similar to 2D twisted bilayers, 3D twisted systems can provide playgrounds for strongly correlated physics. As an example, superconducting instability should be enhanced in 3D twisted graphite near the magic angles due to the strong velocity suppression. In addition to solid state materials, our theory should be realizable in photonic and phononic systems, where 2D Dirac physics and 3D Weyl physics have been demonstrated [24-32]. The flexibility of building metamaterials makes them promising platforms for our proposed
3D topological twistronics. Our predictions can be tested in experimental systems as disparate as suitably designed photonic structures and engineered solid state heterostructures as well as in layered systems made from graphite and manually stacked multilayer graphene.

Acknowledgments. We thank Yang-Zhi Chou for discussions. This work is supported by the Laboratory for Physical Sciences and Microsoft. R.-X.Z. is supported by a JQI Postdoctoral Fellowship.
[1] R. Bistritzer and A. H. MacDonald, Proc. Natl. Acad. Sci. USA 108, 12233 (2011).

[2] S. Carr, D. Massatt, S. Fang, P. Cazeaux, M. Luskin, and E. Kaxiras, Phys. Rev. B 95, 075420 (2017).

[3] Y. Cao, V. Fatemi, S. Fang, K. Watanabe, T. Taniguchi, E. Kaxiras, and P. Jarillo-Herrero, Nature (London) 556, 43 (2018).

[4] Y. Cao, V. Fatemi, A. Demir, S. Fang, S. L. Tomarken, J. Y. Luo, J. D. Sanchez-Yamagishi, K. Watanabe, T. Taniguchi, E. Kaxiras, R. C. Ashoori, and P. Jarillo-Herrero, Nature (London) 556, 80 (2018).

[5] M. Yankowitz, S. Chen, H. Polshyn, Y. Zhang, K. Watanabe, T. Taniguchi, D. Graf, A. F. Young, and C. R. Dean, Science 363, 1059 (2019).

[6] X. Lu, P. Stepanov, W. Yang, M. Xie, M. A. Aamir, I. Das, C. Urgell, K. Watanabe, T. Taniguchi, G. Zhang et al., Nature (London) 574, 653 (2019).

[7] A. L. Sharpe, E. J. Fox, A. W. Barnard, J. Finney, K. Watanabe, T. Taniguchi, M. A. Kastner, and D. GoldhaberGordon, Science 365, 605 (2019).

[8] M. Serlin, C. L. Tschirhart, H. Polshyn, Y. Zhang, J. Zhu, K. Watanabe, T. Taniguchi, L. Balents, and A. F. Young, Science 367, 900 (2020).

[9] P. San-Jose, J. González, and F. Guinea, Phys. Rev. Lett. 108, 216802 (2012).

[10] H. C. Po, L. Zou, A. Vishwanath, and T. Senthil, Phys. Rev. X 8, 031089 (2018).

[11] M. Koshino, N. F. Q. Yuan, T. Koretsune, M. Ochi, K. Kuroki, and L. Fu, Phys. Rev. X 8, 031087 (2018).

[12] J. Kang and O. Vafek, Phys. Rev. X 8, 031088 (2018).

[13] J. Liu, J. Liu, and X. Dai, Phys. Rev. B 99, 155415 (2019).

[14] Z. Song, Z. Wang, W. Shi, G. Li, C. Fang, and B. A. Bernevig, Phys. Rev. Lett. 123, 036401 (2019).

[15] A. A. Soluyanov, D. Gresch, Z. Wang, Q. Wu, M. Troyer, X. Dai, and B. A. Bernevig, Nature (London) 527, 495 (2015).

[16] D. I. Pikulin, A. Chen, and M. Franz, Phys. Rev. X 6, 041021 (2016).

[17] J. M. B. Lopes dos Santos, N. M. R. Peres, and A. H. Castro Neto, Phys. Rev. Lett. 99, 256802 (2007).
[18] See Supplemental Material at http://link.aps.org/supplemental/ 10.1103/PhysRevResearch.2.022010 for a perturbation theory that estimates the first magic angle in chiral twisted graphite, and a derivation of the interlayer tunneling matrix in a twisted Weyl semimetal, which includes Ref. [33].

[19] E. Khalaf, A. J. Kruchkov, G. Tarnopolsky, and A. Vishwanath, Phys. Rev. B 100, 085109 (2019).

[20] T. Cea, N. R. Walet, and F. Guinea, Nano Lett. 19, 8683 (2019).

[21] C. Mora, N. Regnault, and B. A. Bernevig, Phys. Rev. Lett. 123, 026402 (2019).

[22] P. Sutter, S. Wimer, and E. Sutter, Nature (London) 570, 354 (2019).

[23] Y. Liu, J. Wang, S. Kim, H. Sun, F. Yang, Z. Fang, N. Tamura, R. Zhang, X. Song, J. Wen, B. Z. Xu, M. Wang, S. Lin, Q. Yu, K. B. Tom, Y. Deng, J. Turner, E. Chan, D. Jin, R. O. Ritchie et al., Nature (London) 570, 358 (2019).

[24] M. C. Rechtsman, J. M. Zeuner, Y. Plotnik, Y. Lumer, D. Podolsky, F. Dreisow, S. Nolte, M. Segev, and A. Szameit, Nature (London) 496, 196 (2013).

[25] L. Lu, Z. Wang, D. Ye, L. Ran, L. Fu, J. D. Joannopoulos, and M. Soljačić, Science 349, 622 (2015).

[26] B. Yang, Q. Guo, B. Tremain, R. Liu, L. E. Barr, Q. Yan, W. Gao, H. Liu, Y. Xiang, J. Chen et al., Science 359, 1013 (2018).

[27] D. Wang, B. Yang, W. Gao, H. Jia, Q. Yang, X. Chen, M. Wei, C. Liu, M. Navarro-Cía, J. Han et al., Nat. Phys. 15, 1150 (2019).

[28] H. Jia, R. Zhang, W. Gao, Q. Guo, B. Yang, J. Hu, Y. Bi, Y. Xiang, C. Liu, and S. Zhang, Science 363, 148 (2019).

[29] M. Xiao, W.-J. Chen, W.-Y. He, and C. T. Chan, Nat. Phys. 11, 920 (2015).

[30] Z. Yang and B. Zhang, Phys. Rev. Lett. 117, 224301 (2016).

[31] F. Li, X. Huang, J. Lu, J. Ma, and Z. Liu, Nat. Phys. 14, 30 (2018).

[32] W. Dorrell, H. Pirie, S. M. Gardezi, N. C. Drucker, and J. E. Hoffman, Phys. Rev. B 101, 121103(R) (2020).

[33] C. Zheng, R. Hoffmann, and D. R. Nelson, J. Am. Chem. Soc. 112, 3784 (1990). 